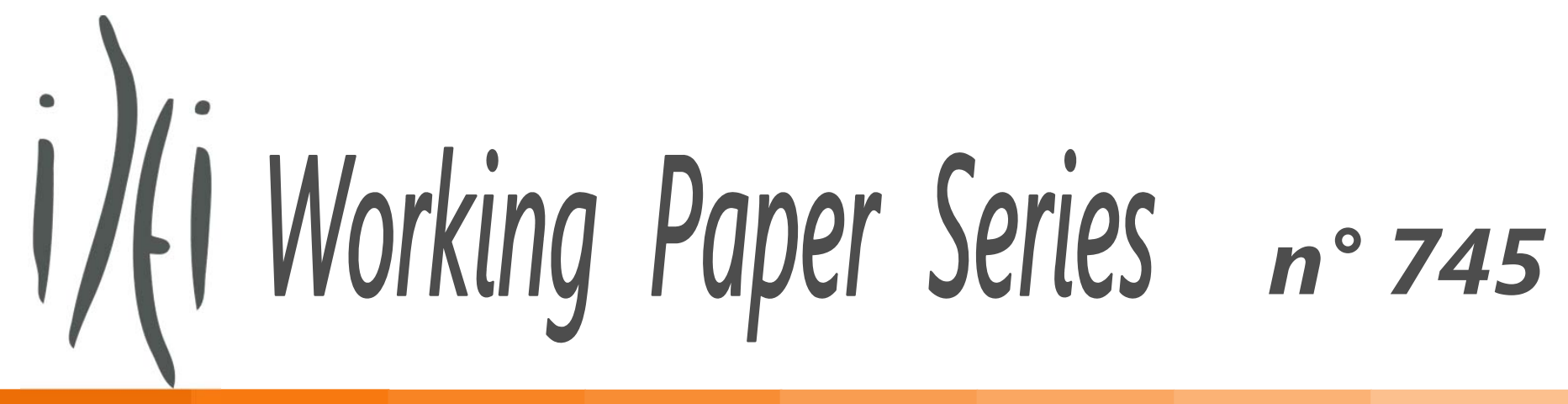

September 2012

« Liberté et société post-utilitariste»

Gilles Saint Paul 


\title{
Liberté et société post-utilitariste
}

\author{
Gilles Saint-Paul*
}

\section{Résumé}

Les fondements utilitaristes d'un gouvernement limité sont instables, dans la mesure où ils supposent des individus rationnels et cohérents. L'hypothèse d'agent rationnel faite par les économistes a récemment été l'objet d'attaques soutenues. L’économie comportementale a suggéré que les individus sont en proie à des biais irrationnels et des incohérences. L'auteur explique comment ces développements ont mené à un post-utilitarisme, qui justifie des interventions paternalistes de l'Etat via des « impôts sur le vice », des interdictions directes ou de nouvelles obligations. La responsabilité individuelle est sévèrement dépréciée, tout comme la confiance dans les marchés. Il conclut que les défenseurs de la liberté individuelle doivent s'éloigner du raisonnement utilitariste, réaffirmer les valeurs fondamentales d'autonomie et de responsabilité, et définir les limites strictes du champ d’intervention du gouvernement.

\begin{abstract}
Utilitarian foundations for limited government are shaky insofar as they assume rational and consistent individuals. Recently economists' assumption of rational actors has come under sustained attack. Behavioural economics has suggested that people are plagued by irrational biases and inconsistencies. The author elucidates how these developments have led to a postutilitarianism which is held to justify paternalistic interventions by the state via 'sin taxes', direct bans or new obligations. Individual responsibility is seriously undermined, as is faith in markets. He concludes that supporters of individual freedom need to move away from utilitarian reasoning, reassert core values of autonomy and responsibility, and define strict limits on the scope of government intervention.
\end{abstract}

Classification JEL : B400, D030, D100, H100

Mots-clés: économie comportementale, utilitarisme, gouvernement, paternalisme

Keywords: behavioural economics, utilitarianism, government, paternalism

\footnotetext{
* Professor of Economics, Toulouse School of Economics
} 


\section{Introduction}

La régulation gouvernementale du comportement privé est de plus en plus acceptée. Le maire de New York a récemment proposé d'interdire la vente de sodas de grande taille dans le cadre d'une croisade contre la «crise » de l’obésité. Dans les médias français, cette information a été présentée sans même un soupçon de critique. Un «expert » fut interviewé, et nous expliqua que cette politique était une bonne idée, car elle pourrait fonctionner. Personne ne se soucia des libertés constitutionnellement protégées, ou ne demanda pourquoi un maire pouvait transgresser de telles libertés à sa discrétion.

Il existe d'innombrables exemples de telles réglementations ${ }^{1}$. De nouvelles sont introduites tous les jours. Elles sont invariablement soutenues par une étude montrant que la mesure proposée aura un effet désirable sur un indicateur statistique clé. Aucune autre question n’est posée, en particulier des questions telles que :

- Pourquoi l’obésité est-elle une « crise »?

- Pourquoi les sodas (qui sont en vente depuis plus d'un siècle) sont-ils responsables de l'obésité d'aujourd'hui, plutôt que les obèses eux-mêmes ?

- Pourquoi le travail du gouvernement consisterait-il à lutter contre l’obésité, en particulier en mettant en place des mesures coercitives qui contraignent tout le monde, que l'on soit obèse ou non?

La pensée libérale traditionnelle, de Locke à Mill, donne des réponses simples à ces questions. Il n’y a aucune raison de s'opposer à l'obésité, tant qu'elle résulte de choix personnels conscients. Que certaines personnes désapprouvent n'est pas une justification de l'intervention du gouvernement qui enfreindrait les droits naturels des individus. Et, finalement, les individus en surpoids peuvent seulement blâmer leurs propres choix passés pour les conséquences négatives qu’ils engendrent, plutôt que la disponibilité de sodas et de sucreries qu'ils auraient pu choisir de ne pas consommer.

La montée sans opposition de politiques paternalistes, telles que la proposition du maire de New York, suggère que nous vivons maintenant dans un paradigme différent de celui des libéraux traditionnels. Comme je l'expose de façon plus détaillée dans mon livre The

\footnotetext{
${ }^{1}$ De nombreux exemples sont présentés par David Harsanyi dans son livre Nanny State (2007).
} 
Tyrannyof Utility (Princeton UniversityPress, 2011), le nouvel Etat paternaliste trouve ses fondements intellectuels dans les développements récents en économie et en d’autres sciences sociales qui offrent des justifications pour beaucoup plus d'intervention publique qu'auparavant.

Alors que la science économique soutenait traditionnellement la liberté des choix individuels et des marchés, ce n’est plus le cas aujourd'hui, car les développements dans le nouveau domaine de l'économie comportementale ont miné les fondements philosophiques et théoriques de ce soutien, et sont au contraire utilisés pour justifier les politiques de plus en plus intrusives qui sont mises en œuvre aujourd’hui.

\section{Economie comportementale et post-utilitarisme}

La notion économique de bien commun trouve historiquement ses racines dans l'utilitarisme. Il signifie que le bien-être de la «société » est défini comme la somme du bien-être (aussi appelé utilité) des individus qui la composent. Pourquoi cette conception mène-t-elle à la présomption que la liberté individuelle est désirable ? Parce ce que la science économique suppose de plus que les individus sont unitaires c'est-à-dire des entités indivisibles dotées de préférences cohérentes- et qu’ils poursuivent rationnellement leur propre intérêt. Sous cette hypothèse, les individus choisissent ce qu'il y a de mieux pour eux parmi l'ensemble des choix possibles : c’est ce que l’on appelle le principe de la préférence révélée. Contraindre leurs choix diminue leur utilité, et ne peut pas, en général, améliorer le bien-être de la société puisqu'il est la somme des utilités individuelles, sauf si la situation d'un autre individu est améliorée d'une quantité supérieure. Cela peut être le cas lorsque nous redistribuons les revenus des riches vers les pauvres, puisqu'il est possible que l'accroissement du bien-être des pauvres soit supérieur à la réduction du bien-être des riches, du point de vue de la société si celle-ci est effectivement utilitariste. Mais cela ne peut pas être le cas si nous interdisons à quelqu'un de boire du soda, puisque cela réduit son bien-être aussi bien que celui des propriétaires des entreprises productrices de soda.

Pourtant, maintenant les économistes préconisent souvent des politiques qui restreignent le comportement individuel telles que les «impôts sur le vice », sur le tabac, les paris ou la nourriture, ou l'obligation de cotiser à des régimes de retraite obligatoires, souvent justifiée par l'argument que si ces régimes étaient facultatifs, de nombreux ménages se retrouveraient dans la pauvreté faute de s'être contraints d'épargner suffisamment lorsqu'ils étaient jeunes. Le 
fait que de plus en plus d'économistes prennent position en faveur de politiques paternalistes traduit une évolution de la pensée économique qui conduit à un abandon progressif de l'utilitarisme traditionnel.

Notons tout d'abord qu'il se pourrait, en principe, que la «société » veuille des gens en meilleure santé que ce que les individus eux-mêmes semblent souhaiter, auquel cas le gouvernement ne maximiserait pas la somme des utilités individuelles, mais poursuivrait plutôt un idéal "supérieur" (ce qui n’est pas sans rappeler les partisans de l’eugénisme dans un passé pas si éloigné). Cette forme de paternalisme repose sur le fait que l'utilité de la "société" n'est pas un agrégat des utilités individuelles. Ce n'est pas là la raison pour laquelle les économistes soutiennent le paternalisme. Ceux-ci continuent à considérer le gouvernement comme en charge du "bien-être social" tel que défini comme somme des bien-être individuels, mais ils abandonnent progressivement la conception de l'individu comme unitaire et rationnel, ce qui implique que le principe de la préférence révélée ne s’applique plus. Et ils le font sur la base de preuves accumulées par les psychologues, les neuroscientifiques, et d'autres, concernant les biais du comportement humain, et qui ont influencé les développements récents de l'économie dite comportementale. Ces biais apparaissent sous des formes variées.

Par exemple, la théorie standard dit que les individus devraient utiliser toute l'information disponible pour construire la meilleure inférence possible sur les paramètres pertinents pour leurs décisions. Mais on observe que les individus traitent souvent l'information de manière à valider leurs choix passés, c'est-à-dire qu'ils donnent moins de poids, voire même ignorent, les signaux qui révèlent que ces choix étaient peut-être erronés. Ce phénomène s’appelle la «dissonance cognitive ${ }^{2}$. Une autre forme de dissonance cognitive est le «biais de disponibilité », par lequel les individus tendent à accorder un poids excessif à leur propre expérience. Ainsi, des individus qui ont été agressés dans un quartier particulier vont inférer que cette zone est généralement dangereuse, alors que ceux qui n’ont pas été agressés continueront à penser que ce même quartier est tout à fait sûr.

D’autres biais suggèrent que l'individu peut être pensé comme étant composé d’identités multiples, plutôt que comme une entité unique et cohérente. Les spécialistes du cerveau ont

\footnotetext{
${ }^{2}$ Pour illustrer cela, Lord, Ross et Lepper (1979) ont réalisé une expérience dans laquelle un groupe d'étudiants est invité à lire les mêmes études sur les effets de la peine de mort. Ils ont montré que les opinions préalables des sujets sont renforcées après la lecture de ces études, indépendamment du fait que ces opinions étaient favorables ou pas.
} 
montré que différents processus mentaux -conscients et inconscients- se font concurrence afin de contrôler les actions de l'individu. Certains de ces processus sont «cognitifs », i.e., commandés par la raison, et d’autres sont « affectifs », i.e., commandés par les émotions et les instincts $^{3}$. Ainsi, les différentes actions entreprises par le même individu sont en fait guidéespar différents processus mentaux. Ce qui signifie qu'elles ne sont pas mutuellement cohérentes et qu'elles peuvent être interprétées comme étant choisies par des identités concurrentes, ou des incarnations concurrentes du même individu.

Le modèle d' « identités multiples » peut être particulièrement pertinent en ce qui concerne les choix intertemporels de l'individu. Si le même individu a différentes incarnations à des dates différentes, alors ses choix intertemporels ne seront plus cohérents, ce qui a d’importantes implications pour de nombreux phénomènes économiques, tels que l'épargne, les incitations, et plus généralement toute transaction ou contrat impliquant différentes dates. Selon le phénomène dit d' "actualisation hyperbolique », le présent immédiat possède un poids particulièrement élevé dans les préférences individuelles, de sorte que l'arbitrage de la consommation entre deux dates n'est pas le même selon le degré d'immédiateté de ces dates. Par exemple, je pourrais décider maintenant d'épargner $100 €$ l'année prochaine, échangeant en fait cette somme contre, disons, $110 €$ l'année suivante. Mais lorsque l'année prochaine arrive, je préfère consommer les $100 €^{4}$. Ainsi, mes choix sont incohérents, comme si la personne qui décide l'année suivante était une incarnation de moi-même différente de celle qui décide aujourd’hui.

Un thème connexe est que les préférences sont déterminées par le contexte dans lequel le choix doit être fait. Les préférences entre deux décisions mutuellement exclusives peuvent dépendre de l'ensemble des autres options ouvertes, ou de la manière dont le problème de choix est formulé, ou des deux. De plus, le bien-être retiré d'une action (disons, consommer un bien) dépend de ce qu'apporte cette action relativement à une référence, en particulier à ce que l'individu en attendait. Cette référence est elle-même influencée par l'expérience économique passée de l’individu, ainsi que par son environnement économique. Par exemple, de nombreuses études empiriques montrent que les choix par défaut ont de l’importance ; plus généralement, la présentation d’un problème de décision -la manière dont il est formulé, en

\footnotetext{
${ }^{3}$ Voir Camereret al. (2005), Berridge (1996) et Ross et al. (1995).

${ }^{4}$ Ce phénomène psychologique fut découvert par Chung et Herrnstein (1967). Il est revenu à la mode en économie, en particulier avec le travail de Laibson (1997).
} 
anglais framing- influence la décision qui sera effectivement prise, pour un ensemble donné de choix possibles.

Un exemple de l'importance des valeurs de référence est l'effet dotation.Il signifie qu'une personne évalue davantage un bien si elle le possède déjà. Thaler (1980) et Kahneman, Knetsch et Thaler (1990) ont par exemple montré que les individus évaluent davantage un bien immédiatement après son acquisition. Dans une expérience, ils ont distribué de manière aléatoire des tasses qui valaient \$5 à leurs cobayes, puis ouvert un marché pour ces tasses. En moyenne, ceux qui avaient reçu lestasses demandaient un prix supérieur à celui offert par ceux qui n’en étaient pas dotés initialement, ce qui suggère que le simple fait de posséder une tasse accroissait leur utilité.

Tous ces biais impliquent qu'on ne peut plus présumer que les individus agissent au mieux de leur intérêt. En outre, si l’on adopte le point de vue selon lequel les individus sont composés d'identités multiples, l'unité de décision de base n'est plus l'individu, mais une unité plus petite : l'incarnation. Par conséquent, le bien-être social utilitariste devrait maintenant être défini comme la somme du bien-être de toutes les incarnations, et non plus des individus. C'est ce que j'appelle le post-utilitarisme. De plus, d'après l'économie comportementale, il n’y a pas de présomption que différentes incarnations au sein du même individu coopèrent entre elles ou, si elles le font, que l'allocation des ressources qui en résulte est en accord avec celle d'un «planificateur social» abstrait. Ce planificateur social pourrait par exemple contraindre les choix de votre incarnation actuelle (en restreignant votre consommation d'alcool ou en vous forçant à épargner une partie de votre revenu) afin d'accroître le bien-être de votre incarnation future.

\section{Les implications du post-utilitarisme}

La transition de l'utilitarisme au post-utilitarisme élimine les garanties contre l'excès de l'intervention du gouvernement qui étaient associées à l'utilitarisme. La présomption que les choix individuels sont irrationnels implique qu'aucune sphère, pas même la sphère privée, n'est à l'abri de l'intervention de l'Etat. En même temps, les politiques publiques et l'éthique publique ne peuvent plus reposer sur la notion de responsabilité individuelle. Discutons de ces deux implications successivement. 
D'après l'utilitarisme traditionnel, le pouvoir politique n'a pas besoin de connaître les préférences des individus. Il peut établir un cadre qui permet de réaliser les transactions mutuellement profitables, sans se soucier du contenu concret de ces transactions. S'il se préoccupe d'inégalité, il peut mettre en œuvre dans ce cadre un mécanisme pour redistribuer les revenus des riches vers les pauvres. Mais, encore une fois, dès que ce mécanisme est en place, il n'a pas à prêter attention à la manière dont l'argent est effectivement utilisé. Du fait du principe de la préférence révélée, la plupart des choix peuvent être abandonnés aux individus, et c'est pourquoi l'utilitarisme aboutit à un préjugé favorable à la liberté des marchés.

Dans la société post-utilitariste, la préférence révélée n’a plus de sens. Cela signifie que ce qui est bon pour les individus doit être mesuré directement, et peut alors leur être imposé, en utilisant une grande variété d’interventions publiques au degré de coercition variable.

Cela implique, par exemple :

1. L’introduction de taxes sur le vice appliquées aux biens considérés comme addictifs ou dangereux pour les personnes qui ont un problème de self-control, tels que l'alcool, le tabac, les armes à feu, les jeux d'argent, les sucreries, les jeux vidéo, la pornographie. Il ya d'innombrables exemples de tels impôts.

2. L’interdiction pure et simple de transactions basées sur le prix convenu ou la nature du bien, pour la raison que peut-être l'une des parties ne peut pas raisonnablement en tirer un bénéfice. Par exemple, une loi française interdit la location de pièces de moins de neuf mètres carrés, alors que personne n’a jamais été obligé de vivre dans ces pièces avant la loi $^{5}$ (inutile de dire que les médias se sont plaints du manque de logement étudiant dans l'année qui a suivi).

3. Manipuler la manière dont les choix sont présentés afin d'utiliser les effets de contexte pour inciter les individus à faire le «bon » choix. Ainsi, les post-utilitaristes recommandent que les gens adhèrent à des fonds de pension comme option par défaut, ou mettent ostensiblement en avant les aliments sains ${ }^{6}$. Bien que cela ait été appelé « paternalisme libertarien », puisqu'il n’y a pas réduction de l'ensemble des choix disponibles, c’est en fait un petit impôt sur les « mauvais » choix (car ceux qui veulent

\footnotetext{
${ }^{5}$ Il est facile de construire des modèles économiques formels pour justifier de telles régulations. Voir par exemple Akerlof et Dickens (1982), Sheshinski (2002), Saint-Paul (2002).

${ }^{6}$ Voir Thaler et Sunstein (2003,2008). Le travail populaire de ces auteurs (Thaler et Sunstein, 2008) a apparemment influencé le premier ministre britannique David Cameron.
} 
faire ce choix doivent consacrer plus de temps et d'attention afin de s'affranchir de l’option par défaut), quelque peu similaire au monopole de l'Etat suédois sur la vente d’alcool qui augmente artificiellement la distance entre les points de vente et réduit les heures d'ouverture. Dans tous ces exemples, nous sommes confrontés à ce qui est en fait un impôt sur le vice en nature plutôt qu'un impôt monétaire. Les travaux de Thaler et Sunstein sur le paternalisme libertarien ont influencé le programme britannique Nudge mis en place par David Cameron qui se propose explicitement de manipuler les processus inconscients des personnes afin de les induire à adopter des comportements plus vertueux ${ }^{7}$.

4. Manipuler les croyances et l'information afin de provoquer la réaction correcte des individus. Cela peut se traduire par la surestimation des risques et des conséquences des comportements à risque dans les campagnes publiques contre le SIDA ou l’obésité ; par leur sous-estimation afin d'éviter des réactions de panique irrationnelles (c’est ce qu’a fait le gouvernement français en 1986 lorsqu'il a nié que le nuage de Tchernobyl ait survolé le pays); par la mise en œuvre d'actions coûteuses pour refreiner les peurs irrationnelles ${ }^{8}$.

5. Enfin, dans une société post-utilitariste, il n’y a pas de présomption de respect des préférences des individus, puisqu'elles ne produisent pas des choix optimaux ou cohérents. On peut donc envisager d'entraîner les gens à être heureux, en concevant par exemple les programmes scolaires à cet effet, comme le conseillent certains auteurs ${ }^{9}$.

Un rôle très important est attribué aux « experts » qui ont la charge de mesurer les effets des diverses politiques gouvernementales sur le bien-être social. En effet, comme le suggère l’exemple donné au début de cet article, nous sommes inondés par les opinions de ces experts dans les médias chaque fois qu'une nouvelle politique visant à restreindre la liberté individuelle est mise en place. Et la liberté individuelle n’a pas de poids dans leur analyse, car les approches utilitariste et post-utilitariste sont toutes deux conséquentialistes. Une politique donnée est évaluée sur la base de son effet final sur la seule allocation matérielle des ressources, sans se préoccuper de savoir si le processus par lequel cette allocation est atteinte

\footnotetext{
${ }^{7}$ Voir par exemple http://www.guardian.co.uk/politics/2010/nov/12/david-cameron-nudge-unit

${ }^{8}$ Voir Salanié et Treich (2007).

${ }^{9}$ Voir Layard (2007).
} 
est acceptable ou non. Dès que les experts ${ }^{10}$ ont fourni la preuve que la politique considérée améliore le bien-être social, elle peut être mise en œuvre sans aucune autre discussion. En particulier, aucune attention n'est portée au fait que la mesure politique affecte différemment les individus. Par exemple, interdire les sodas peut empêcher les buveurs compulsifs de prendre du poids, mais qu'en est-il des buveurs raisonnables qui n’ont pas de problème particulier de poids et dont l'ensemble de choix est limité de manière coercitive ? Cet argument n'importe pas aux yeux des paternalistes, non seulement parce qu'ils sont conséquentialistes, mais aussi parce que, en tant que post-utilitaristes, ils ne se préoccupent que du bien-être total sans tenir compte des cas individuels ${ }^{11}$. Aussi longtemps que nous pensons qu'en moyenne, la mesure politique fonctionne (par exemple, si nous avons prouvé que le poids moyen des New-Yorkais baissera si les sodas supersized sont interdits), elle sera considérée comme bénéfique. Cela signifie à son tour qu'il est suffisant de mesurer l'effet moyen de la politique, et de mener toute la discussion en termes de moyennes.

Pourtant, même dans une perspective post-utilitariste, il semblerait plus efficace de laisser les individus sans problème de poids consommer ce qu'ils veulent, et d'imposer des restrictions seulement aux personnes en surpoids. Par exemple, pourquoi ne pas leur faire payer plus d'impôts,ce qui les inciterait à mincir ? Cela serait probablement plus efficace qu'une taxe sur les sodas ou une interdiction des sodas, puisque, avec un tel mécanisme, toutes les sources d'excès de poids seraient imposées de la même manière. On pourrait objecter que cela poserait un problème d'égalité. Peut-être, mais toute croisade contre l'obésité imposera inévitablement des coûts supérieurs à ceux enclins à manger plus, et on peut donc affirmer que de telles politiques sont intrinsèquement inégalitaires. Une autre objection est qu'elle serait impraticable, puisqu'elle impliquerait d'obtenir le poids d'une personne pour déterminer son impôt sur le revenu. Cependant, cela n’est pas différent de nombreux autres éléments nécessaires à la déclaration d’impôts, pour lesquels les autorités fiscales collectent des informations sur la base d'une déclaration volontaire sujette à contrôle et sanction. La véritable raison pour laquelle taxer directement le poids n'est pas envisagé est que le paradigme post-utilitariste considère que de tels programmes d'incitations et, plus généralement, les politiques qui reposent sur la responsabilité individuelle, sont inefficaces et manquent de justification morale.

\footnotetext{
${ }^{10}$ Ces experts peuvent bien évidemment être eux-mêmes sujets aux biais, bien que cette possibilité soit fréquemment ignorée.

${ }^{11}$ C'est aussi vrai, bien sûr, pour l’utilitarisme.
} 
Il n’est sensé de tenir les individus pour responsables des conséquences de leurs actions que s’ils sont considérés comme unitaires. Si nous les considérons au contraire comme consistant en identités multiples, il s'ensuit que l'incarnation qui supporte les conséquences d'une action peut être différente de celle qui a choisi cette action. Ainsi, si les individus souffrent des conséquences de leurs choix passés, nous les considérons comme étant victimes de leurs incarnations passées comme s’ils étaient des individus différents, qui ne peuvent pas être pénalisés puisqu'ils n’existent plus.

Dans un monde post-utilitariste, il n'est plus moralement justifié d'imposer des sanctions négatives aux gens du fait de leurs choix passés. Et, par la même occasion, s’appuyer sur les structures traditionnelles de récompenses et de sanctions peut être inefficace, puisque les incitations produites par ces structures sont affaiblies par le fait que l'incarnation qui est punie/récompensée sera différente de celle qui entreprend l'action. Ainsi, il est difficile de dissuader Mr Hyde de commettre un crime s'il pense que Dr Jekyll, et non pas lui-même, subira la condamnation qui s'en suivra.

Vues sous cet angle, il n’est pas surprenant que nos sociétés reposent de plus en plus sur la prévention plutôt que sur la sanction. L'accent mis sur la prévention ouvre en retour la porte à l'atteinte illimitée du gouvernement aux libertés individuelles. Dans la société libérale traditionnelle, seules les actions qui nuisent directement à d'autres personnes, telles que le meurtre, la violence et le vol, sont censées être pénalisées. Dans la société post-utilitariste, où les gens sont considérés comme incapables de self-control, toute action qui peut déclencher une chaîne d'événements dont les conséquences finales sont nuisibles peut être légitimement interdite. Puisque l'incarnation, et non plus l'individu, est désormais l'unité élémentaire de l'analyse du bien-être, la même logique s’applique, que la nuisance faite à la fin de la chaîne d’événements soit subie par les individus eux-mêmes ou par des tiers.

Ainsi les cigarettes en chocolat ont été interdites en France, au prétexte qu'elles peuvent inciter les enfants à fumer plus tard (bien que ni les cigarettes, ni le chocolat ne soient interdits). Le maire de la ville de New York veut interdire les sodas de grande taille, car certaines personnes peuvent en consommer trop et devenir obèses. Ces mesures préventives sont destinées à protéger les individus contre eux-mêmes. D’autres mesures, telles qu'une loi interdisant aux barmen en France de servir de l'alcool aux clients qui peuvent être ivres, et ce 
contre la volonté du client et contre leur propre intérêt commercial, sont censés éviter que des tiers subissent des préjudices, comme par exemple dans un accident de la route.

Au fur et à mesure que de nouvelles mesures sont introduites, et pour la même raison, nous voyons de plus en plus de réticence à laisser les individus supporter les conséquences de leurs propres choix. Ceci explique pourquoi nous observons une assistance sociale inconditionnelle, couplée à des contributions obligatoires aux systèmes de retraite ; des traitements médicaux gratuits pour les maladies causées par certains modes de vie couplés à des taxes sur le tabac, des prix minimaux pour l'alcool en Ecosse et au Danemark, des taxes sur les aliments contenant des graisses saturées ; l'accès gratuit à la méthadone et aux seringues pour les toxicomanes, alors que la consommation de drogue est illégale pour les non-toxicomanes.

Remplacer la répression par la prévention implique néanmoins une contradiction. Pour faire respecter la prévention, le gouvernement doit faire passer de nouvelles lois qui rendent illégales des actions qui ne l'étaient pas auparavant. Mais, pour faire respecter ces lois, il doit mettre en place des sanctions à l'encontre de ceux les enfreignent. Ainsi, nous ne pouvons pas nous passer de la répression. Mais si la loi préventive est adoptée au motif que la répression ne fonctionne pas, comment cette loi peut-elle être exécutée ? La réponse à cette contradiction est d’appliquer de manière sélective les outils répressifs aux seuls individus et organisations responsables. De plus, il est efficace pour un Etat post-utilitariste d'attribuer à ces entités responsables la tâche de faire respecter les mesures préventives pour contraindre le comportement des individus irresponsables, lorsque c'est réalisable. Ainsi, dans l'exemple précédent, on ne peut pas faire confiance au buveur irresponsable, et on demande au barman d'internaliser le bien-être social en refusant de servir son client. C'est le phénomène de transfert de responsabilité.

En un sens, le transfert de responsabilité est un nouveau socialisme de la rationalité. Comme dans une société marxiste où la société donne à chacun selon ses besoins et demande à chacun selon ses capacités, dans un monde où le transfert de responsabilité est omniprésent, ceux que l'on peut considérer comme rationnels portent le poids de la responsabilité. Les incitations et les sanctions ne s'appliquent qu'à eux, alors que les autres sont traités comme étant moralement et légalement mineurs. Comme dans l'exemple du barman et bien d'autres, les sanctions pour leurs actions nuisibles sont transférées à la caste responsable, dans la mesure où ce sont leurs interactions avec ces responsables qui peuvent être considérées comme cause 
de l'action nuisible. Et, comme dans une société marxiste où il n'y a pas d'incitation à créer de la richesse, il en résulte des effets pervers. Les individus peuvent volontairement se mettre dans des situations où ils sont exemptés de responsabilité individuelle, alors que les individus responsables peuvent s’abstenir d'interagir avec la caste irresponsable ${ }^{12}$.

Un autre domaine exposé à une régulation croissante du fait des préoccupations paternalistes, est celui des marchés. Les marchés sont l’ennemi des paternalistes pour au moins trois raisons. Premièrement, on ne peut plus présumer qu'ils équilibreront les échanges de façon efficace, car les agents qui réalisent des arbitrages ne sont plus vus comme agissant rationnellement, mais plutôt comme étant influencés par des biais comportementaux, tels que l'excès de confiance ou le trading compulsif. Dès lors, on ne peut plus présumer que le prix d'équilibre de marché soit correct. Deuxièmement, les vendeurs peuvent exploiter les biais des acheteurs, en utilisant par exemple des prix d'appel ou différentes formes de manipulation publicitaire $^{13}$, afin de les inciter à acheter contre leur propre intérêt. Troisièmement, les marchés peuvent permettre aux individus de s’affranchir des politiques paternalistes qui leur sont imposées. L’interdiction des sodas de grande taille n'aura aucun effet s'il est possible d'acheter deux sodas de taille normale. Les plans d'épargne obligatoire peuvent être contournés en empruntant et en remboursant les emprunts avec les prestations que verseront ces plans. Etre interdit de casino, même volontairement, ne peut pas être respecté si de nouveaux casinos entrent constamment sur le marché, ou si les sites de pari en ligne prolifèrent.

\section{Conclusion}

Pour ceux d'entre nous qui pensent que la liberté individuelle et une intervention publique limitée sont d'importantes pierres angulaires de notre société, y’a-t-il un moyen de résister à la montée de l’Etat paternaliste?

Il serait tentant de restaurer les anciens fondements utilitaristes d'un gouvernement limité en niant la pertinence des biais comportementaux. Néanmoins, ces biais sont abondamment

\footnotetext{
${ }^{12}$ Il existe d'innombrables exemples de réglementations qui se retournent contre ceux qu'elles sont supposées protéger. Par exemple, en France, il est très difficile d'expulser un locataire pour le non-paiement du loyer s'il est au chômage ou âgé. Il en résulte que les personnes âgées ou les gens ayant un travail précaire éprouvent des difficultés considérables pour louer un logement.

${ }^{13}$ Ces techniques sont ironiquement les mêmes que celles que le programme Nudge cité plus haut se propose d'utiliser afin de promouvoir les comportements vertueux.
} 
démontrés dans les travaux récents de recherche, et les attaques contre la liberté individuelle que nous observons montrent à quel point ces fondements étaient fragiles ${ }^{14}$.

Il est certain que la raison pour laquelle nous nous soucions des droits individuels va au-delà de l'hypothèse qu'ils sont un outil efficace pour décentraliser l'allocation des ressources. Il est dès lors nécessaire d'aller au-delà de l'utilitarisme et de rétablir l'autonomie individuelle, la liberté et la responsabilité comme les valeurs fondamentales de notre société et de définir en conséquence les limites strictes de l'étendue des interventions gouvernementales. Cela implique que nous devons vivre avec les conséquences et nous débarrasser de l’idée que le gouvernement est un tuteur bienveillant en charge du bonheur général. A l'heure actuelle, malheureusement, peu de forces semblent s’opposer à la montée du paternalisme.

\footnotetext{
${ }^{14}$ Notons cependant que des arguments post-utilitaristes contre le paternalisme peuvent être avancés, en remarquant que les politiciens et les bureaucrates sont eux-mêmes sujets aux biais comportementaux. Voir Gleaser (2005).
} 


\section{Bibliographie}

G. Akerlof et T. Dickens[1982]: The Economic Consequences of Cognitive Dissonance, American Economic Review, 72(3),pp. 307-319.

K. Berridge [1996] : Food Reward: Brain Substrates of Wanting and Liking, Neuroscience and Biobehavioral Reviews, 20(1), pp. 1-25.

C. Camerer, G. Loewenstein et D. Prelec[2005] : Neuroeconomics: How Neuroscience Can Inform Economics, Journal of Economic Literature,43(1), pp. 9-64.

S.H. ChungetR.J. Herrnstein [1967] : Choice and Delay of Reinforcement, Journal of the Experimental Analysis of Behavior, 10(1), pp. 67-74.

E. Glaeser [2005] : Paternalism and Psychology, Harvard Institute of Economic Research Discussion Paper.

D. Harsanyi [2007] : Nanny State,Broadway Books.

D. Kahneman, J. Knetsch et R. Thaler[1990] Experimental Tests of the Endowment Effect and the Coase Theorem, Journal of Political Economy, 98(6), pp. 1325-1348.

D. Laibson [1997] : Golden Eggs and Hyperbolic Discounting, Quarterly Journal of Economics, 112(2), pp. 443-477.

R. Layard [2007] : Happiness and the Teaching of Values. Centre Piece(summer),pp. 18-23.

LordCharlesG., L. Ross et M.R. Lepper [1979] : Biased Assimilation and Attitude Polarization: The Effects of Prior Theories on Subsequently Considered Evidence, Journal of Personality and Social Psychology, 37(11), pp. 2098-2109. 
L. Ross, M.R. Lepper et M. Hubbard[1995] : Perseverance in Self-Perception and Social Perception: Biased Attributional Processes in the Debriefing Paradigm, Journal of Personality and Social Psychology,32(5), pp. 880-892.

G. Saint-Paul [2002] : Cognitive Ability and Paternalism, Centre for Economic Policy Research Working Paper No. 3642.

G. Saint-Paul [2011] : The tyranny of utility, Princeton University Press

F. Salanié et N. Treich [2007] : Regulation in Happyville, Economic Journal,119(4), pp. 665-679.

E. Sheshinski [2002] : Bounded Rationality and Socially Optimal Limits on Choice in a SelfSelection Model, Hebrew University Working Paper No.330.

R. Thaler [1980] : Toward a Positive Theory of Consumer Choice, Journal of Economic Behavior and Organization,1(1), pp. 39-60.

R. Thaler et C.R. Sunstein [2003] : Libertarian Paternalism, American Economic Review,93(2), pp. 175-179.

R. Thaler et C.R. Sunstein [2008] : Nudge: Improving Decisions about Health Wealth and Happiness, Yale University Press. 